\title{
RNAi-Mediated Antiviral Approach to SARS-CoV-2
}

\author{
P Shaik Syed Ali ${ }^{1}$, A Sheeza ${ }^{1}$ and J Jasmine ${ }^{2 *}$ \\ ${ }^{1}$ School of Medicine, Maldives National University, Male', Maldives \\ ${ }^{2}$ DKM College for Women, Vellore, Tamil Nadu, India \\ *Corresponding Author: J Jasmine, DKM College for Women, Vellore, Tamil Nadu, \\ India.
}

\author{
Received: May 21, 2020 \\ Published: June 16, 2020 \\ (C) All rights are reserved by J Jasmine., \\ et al.
}

\begin{abstract}
In 2003, severe acute respiratory syndrome (SARS) caused by SARS coronavirus (CoV) affected 26 countries with 8000 cases. The coronavirus disease 2019 (COVID-19) caused by SARS-CoV-2 was first reported in Wuhan, China in December 2019. It rapidly evolved into a pandemic disease resulting in an unprecedented health crisis. Antiviral drugs and vaccines against SARS-CoV-2 and related coronaviruses are crucial to prevent any future epidemics and pandemics. RNA interference (RNAi), an RNA guided post transcriptional gene silencing mechanism, plays important role in viral defense in mammals including humans. RNAi can inhibit the virus replication and expression of viral proteins through the leverage of small interfering RNAs (siRNAs). Therefore, RNAi an innate viral defense mechanism distributed in human cells might be a potential antiviral approach to SARS-CoV-2.

Keywords: Antiviral; COVID-19; RNAi; SARS-CoV-2; siRNAs
\end{abstract}

\section{Abbreviations}

ACE-2: Angiotensin Converting Enzyme 2; Ago: Argonaute; CoV: Coronavirus; COVID-19: Coronavirus Disease 2019; DGCR8: Di-George Syndrome Critical Region 8 Gene; ds: Double Stranded; GTP: Guanosine Triphosphate; mRNA: Messenger RNA; miRNAs: microRNAs; nt: Nucleotides; pre-miRNA: Precursor miRNA; priRNA: Primary Hairpin RNA; RNA: Ribonucleic Acid; RNAi: RNA Interference; RISC: RNA Induced Silencing Complex; RSV: Respiratory Syncytial Virus; SARS: Severe Acute Respiratory Syndrome; siRNAs; Small Interfering RNAs; VSR: Viral Suppressor of RNAi.

\section{Introduction}

Survival of humans against infectious diseases is crucially dependent on a competent immune system that eliminates microbial infections including viruses, which are intracellular pathogens. Among the innate immunity pathways, RNAi plays a robust role in antiviral defense in mammals [1,2]. RNAi pathway inhibits the viral replication through the production of siRNAs [3,4]. One of the strands of siRNA associates with Argonaute (Ago) protein to base pair perfectly to its target RNA and subsequently cleaves the cellular messenger RNA (mRNA) or viral RNA [5,6]. In addition, microRNAs (miRNAs) that are central molecules of RNAi in regulating the gene expression have been implicated in antiviral defense [7]. RNAi technology is used by the researchers worldwide to identify specific gene functions. RNAi-based drugs for the prevention and treatment of human diseases notably viral infections and cancers are under development $[8,9]$.
Innate RNAi machinery distributed in human cells can be exploited to specifically target the viral RNA and inhibit the expression of viral proteins [10]. Therefore, the RNAi breakthrough technology can be a significant tool to protect humans from viral infections. This mini-review aims to provide insights into RNAi as a potential antiviral approach to SARS-CoV-2 infection.

\section{SARS-CoV-2}

SARS-CoV-2 is an enveloped, positive sense single stranded RNA virus belonging to the genus Betacoronavirus [11]. COVID-19, a rapidly spreading pandemic disease, is caused by SARS-CoV-2. Genome analysis of SARS-CoV-2 revealed it to have a close genetic identity with SARS-CoV, which caused the 2003 epidemic with approximately 8000 cases $[11,12]$. The receptor binding domain of spike glycoprotein (S) of SARS-CoV-2 and SARS-CoV is structurally homologous to each other despite having some non-identical residues. The structural homology of the S-protein suggested the possibility that SARS-CoV-2 may recognize the same receptor similar to SARS-CoV, the human angiotensin converting enzyme 2 (ACE2 ) receptor, that was later confirmed $[13,14]$. On the other hand SARS-CoV nucleocapsid (N) protein, which is a viral suppressor of RNAi (VSR), has nearly $90 \%$ amino acid identity with SARS-CoV-2 $\mathrm{N}$-protein. This indicates that SARS-CoV-2 N-protein may act as a VSR $[15,16]$ that corroborates the possibility of SARS-CoV-2 inhibition by siRNAs in eukaryotic cells. 
Mechanism of RNAi machinery

RNAi is an evolutionarily conserved RNA guided gene silencing mechanism found in eukaryotes. RNAi acts at the posttranscriptional level specifically triggered by double stranded (ds) RNAs. The fundamental molecules required to execute the process of RNAi are siRNAs and miRNAs. miRNAs are approximately $22 \mathrm{nu}-$ cleotide (nt) long noncoding RNAs found in eukaryotes $[17,18]$. The miRNA biogenesis is a multistep process that occurs in the nucleus and cytoplasm. The primary hairpin RNA (pri-RNA) is cleaved by the Drosha and DGCR8 (Di-George syndrome critical region 8 gene) to 70nt long hairpin precursor miRNA (pre-miRNA) $[18,19]$. Exportation of pre-miRNA from the nucleus to cytoplasm occurs through Exportin5:RanGTP, a membrane protein complex [20]. In the cytoplasm, the enzyme Dicer cleaves the pre-miRNA to 21-25nt long miRNAs. One of the strands of miRNA, the passenger strand, is cleaved leaving the miRNA* guide strand to bind the RNA induced silencing complex (RISC) [19]. The chief catalytic unit in RISC is Ago protein. RISC with the loaded miRNA seeks the target mRNA resulting in cleavage of mRNA or repression of protein translation [21].

Introduction of exogenous dsRNAs result in the generation of siRNAs by Dicer that are 21 - 25nt long with 3' 2nt overhangs [22]. One of the strands of siRNA incorporates into RISC and cleaves the target mRNA [23]. Similarly, during viral infection, the dsRNA of the viruses are cleaved by Dicer to generate siRNAs. RISC loaded with the siRNA seeks and specifically cleaves the complementary viral RNA [24].

Inhibition of respiratory viruses by siRNAs mediated by RNAi

Recent studies demonstrated the ability of synthetic siRNAs to inhibit the production of viruses in vivo. Influenza virus, that causes frequent epidemics, infects the respiratory tract. siRNAs have been able to efficiently inhibit the production of influenza virus in cultured cells post infection $[25,26]$. The fusion protein, responsible for syncytium formation, in respiratory syncytial virus (RSV) was inhibited efficiently by siRNA mediated interference shown by the absence of syncytium in virus infected cells [27].

Additionally, siRNA was demonstrated to be an efficient antiviral for SARS-CoV by its ability to inhibit several of its gene products. siRNA blocked the replication of SARS-CoV in Vero cells by specific inhibition of viral RNA polymerase [28]. The cytopathic effects on Vero cells were reduced if the siRNAs were introduced before infecting the cells with SARS-CoV [29]. Remarkably, siRNAs could suppress the S-genes of SARS-CoV that encodes the S-proteins [30], which binds ACE-2 receptors in human cells $[13,14]$.
SARS-CoV-2 shares approximately $80 \%$ nucleotide sequence identity with SARS-CoV [31]. Notably, the S-protein of SARS-CoV-2 shares $80 \%$ amino acid sequence identity with SARS-CoV [32]. Therefore, it is very likely that the siRNA based antiviral application outcomes on SARS-CoV could be extrapolated on SARS-CoV-2.

siRNA can be administered either locally or systemically. However, nucleases specifically RNAse tends to break down the unprotected RNAs. In order to enhance the siRNA stability and increase cellular uptake several approaches have been developed. Techniques involving chemical modifications of ribose sugar or nucleotide bases are widely used [33]. Moreover, chemical modifications of siRNA prevent the host to mount immune response against it [34]. Administration of siRNAs through inhalation is a possible approach for respiratory viruses. In animal models, siRNA administration through nasal mucosa inhibited the RSV infection and other respiratory viruses $[27,35]$. In order to achieve an efficient prophylaxis, the siRNAs must be administered before the virus infection. Based on the above research studies on SARS and other respiratory viruses, it is persuasive that RNAi mediated interference of SARSCoV-2 replication and expression of proteins might be a potential and specific antiviral approach.

\section{Conclusion}

COVID-19 caused by SARS-CoV2 started as an epidemic in South China and has since evolved into a pandemic due to lack of effective antiviral or vaccines. There is an immediate need of effective antiviral approaches in order to control the SARS-CoV-2 or other coronavirus related future epidemics and pandemics. Currently, vaccine for SARS-CoV-2 is not available due to novelty of the virus. A key advantage of using siRNA based RNAi mediated antiviral approach to SARS-CoV-2 over vaccines is because it is an innate viral defense mechanism widely distributed in all mammalian cells including humans. The other benefits are low cost, relatively easy to design and more importantly no off target effects.

\section{Conflict of Interest}

The authors declare that they have no conflicts of interest.

\section{Bibliography}

1. Berkhout B. "RNAi-mediated antiviral immunity in mammals". Current Opinion in Virology 32 (2018): 9-14.

2. Li Y., et al. "RNA interference functions as an antiviral immunity mechanism in mammals". Science 342.6155 (2013): 231234. 
3. Jeang KT. "RNAi in the regulation of mammalian viral infections". BMC Biology 10 (2012): 58.

4. Zamore PD., et al. "RNAi: double-stranded RNA directs the ATP-dependent cleavage of mRNA at 21 to 23 nucleotide intervals". Cell 101.1 (2000): 25-33.

5. MacRae IJ., et al. "In vitro reconstitution of the human RISCloading complex". Proceedings of the National Academy of Sciences of the United States of America 105.2 (2008): 512-517.

6. Wilson RC and Doudna JA. "Molecular mechanisms of RNA interference". The Annual Review of Biophysics 42 (2013): 217239.

7. Vijayendran D., et al. "Arthropod viruses and small RNAs". Journal of Invertebrate Pathology 114.2 (2013): 186-195.

8. Dave RS and Pomerantz RJ. "RNA interference: on the road to an alternate therapeutic strategy!". Reviews in Medical Virology 13.6 (2003): 373-385.

9. Yin JQ., et al. "siRNA agents inhibit oncogene expression and attenuate human tumor cell growth". Journal of Experimental Therapeutics and Oncology 3.4 (2003): 194-204.

10. Gitlin L., et al. "Short interfering RNA confers intracellular antiviral immunity in human cells". Nature 418.6896 (2002): 430-434.

11. Lu R., et al. "Genomic characterisation and epidemiology of 2019 novel coronavirus: implications for virus origins and receptor binding". Lancet 395.10224 (2020): 565-574.

12. Jiang S., et al. "An emerging coronavirus causing pneumonia outbreak in Wuhan, China: calling for developing therapeutic and prophylactic strategies". Emerging Microbes and Infections 9.1 (2020): 275-277.

13. Shang J., et al. "Structural basis of receptor recognition by SARS-CoV-2". Nature 581.7807 (2020): 221-224.

14. Ou X., et al. "Characterization of spike glycoprotein of SARSCoV-2 on virus entry and its immune cross-reactivity with SARS-CoV". Nature Communications 11.1 (2020): 1620.

15. Kannan S., et al. "COVID-19 (Novel Coronavirus 2019) - recent trends". European Review for Medical and Pharmacological Sciences 24.4 (2020): 2006-2011.
16. Cui L., et al. "The nucleocapsid protein of coronaviruses acts as a viral suppressor of RNA silencing in mammalian cells". Journal of Virology 89.17 (2015): 9029-9043.

17. Ambros V. "The functions of animal microRNAs". Nature 431.7006 (2004): 350-355.

18. Gregory RI., et al. "MicroRNA biogenesis: isolation and characterization of the microprocessor complex". Methods in Molecular Biology 342 (2006): 33-47.

19. Lee Y., et al. "The nuclear RNase III Drosha initiates microRNA processing". Nature 425.6956 (2003): 415-419.

20. Bohnsack MT., et al. "Exportin 5 is a RanGTP-dependent dsRNA-binding protein that mediates nuclear export of pre-miRNAs". RNA 10.2 (2004): 185-191.

21. Kupferschmidt K. "A lethal dose of RNA". Science 341.6147 (2013): 732-733.

22. Bernstein E., et al. "Role for a bidentate ribonuclease in the initiation step of RNA interference". Nature 409.6818 (2001): 363-366.

23. Lagana A., et al. "Computational design of artificial RNA molecules for gene regulation". Methods in Molecular Biology 1269 (2015): 393-412.

24. Rawlings RA., et al. "Viral RNAi suppressor reversibly binds siRNA to outcompete Dicer and RISC via multiple turnover". Journal of Molecular Biology 408.2 (2011): 262-276.

25. Ge Q., et al. "RNA interference of influenza virus production by directly targeting mRNA for degradation and indirectly inhibiting all viral RNA transcription". Proceedings of the $\mathrm{Na}$ tional Academy of Sciences of the United States of America 100.5 (2003): 2718-2723.

26. Kapadia SB., et al. "Interference of hepatitis C virus RNA replication by short interfering RNAs". Proceedings of the National Academy of Sciences of the United States of America 100.4 (2003): 2014-2018.

27. Bitko V., et al. "Inhibition of respiratory viruses by nasally administered siRNA". Nature Medicine 11.1 (2005): 50-55.

28. He ML., et al. "Inhibition of SARS-associated coronavirus infection and replication by RNA interference". The Journal of the American Medical Association 290.20 (2003): 2665-2666. 
29. Zhang R., et al. "Inhibiting severe acute respiratory syndromeassociated coronavirus by small interfering RNA". Chinese Medical Journal 116.8 (2003): 1262-1264.

30. Zhang Y., et al. "Silencing SARS-CoV spike protein expression in cultured cells by RNA interference". FEBS Letters 560.1-3 (2004): 141-146.

31. Zhou Y., et al. "Network-based drug repurposing for novel coronavirus 2019-nCoV/SARS-CoV-2". Cell Discovery Journal 6 (2020): 14.

32. Walls AC., et al. "Structure, function and antigenicity of the SARS-CoV-2 spike glycoprotein". Cell 181.2 (2020): 281-292.

33. Kaczmarek JC., et al. "Advances in the delivery of RNA therapeutics: from concept to clinical reality". Genome Medicine 9.1 (2017): 60.

34. Broering R., et al. "Chemical modifications on siRNAs avoid Toll-like-receptor-mediated activation of the hepatic immune system In vivo and In vitro". International Immunology 26.1 (2014): 35-46.

35. Alvarez R., et al. "RNA interference-mediated silencing of the respiratory syncytial virus nucleocapsid defines a potent antiviral strategy". Antimicrobial Agents and Chemotherapy 53.9 (2009): 3952-3962.

\section{Assets from publication with us}

- Prompt Acknowledgement after receiving the article

- Thorough Double blinded peer review

- Rapid Publication

- Issue of Publication Certificate

- High visibility of your Published work

Website: https://www.actascientific.com/

Submit Article: https://www.actascientific.com/submission.php Email us: editor@actascientific.com

Contact us: +919182824667 